\section{Concentration of Heavy Water by Spontaneous Evaporation}

Ir has been stated that deuterium can be concentrated much more efficiently by the spontaneous atmospheric evaporation of water than by boiling ${ }^{1}$. This suggestion is based on an experiment in which Poznan water, by spontaneous evaporation to $1 / 40$ of its volume, gave a residue claimed to contain $1 \cdot 65$ per cent of deuterium. The experiment would appear to be incorrect, however, for even assuming 'semipermeable membrane' separation $(\alpha=\infty)$ the proportion of deuterium in Poznan water would, according to this experiment, have the highly improbable value $1 / 2,500$. Of course, the separation coefficient, $\alpha$, cannot be infinity : under ideal conditions it could amount to the isotopic vapour pressure ratio. Taking $\alpha=1 \cdot 15$ as an outside figure, the deuterium content of Poznan water, according to the reported evaporation experiment, becomes $1 / 100$.

University College,

E. D. Hughes.

C. K. INGOLD.

C. L. Wrison.

London.

July 13.

${ }^{2}$ T. Tucholski, Nature, 134, 29, July 7, 1934.

\section{Constitution of Vasicine}

SPATH and Nikawitz ${ }^{1}$ have investigated the behaviour of a base, $\mathrm{C}_{11} \mathrm{H}_{12} \mathrm{ON}_{2}$, termed peganine, which the firm of E. Merck (Darmstadt) isolated from the mother liquors of the alkaloids of Peganum harmala. A little later, Späth and Kuffner ${ }^{2}$ found that peganine is identical with the base vasicine isolated from Adhatoda vasica, Nees, by Sen and Ghose $^{3}$ and more recently studied by Ghose, Krishna, Narang and Rây ${ }^{4}$; the name peganine therefore becomes superfluous.

The formulation of vasicine by Spath and Nikawitz as 4-hydroxy-3-allyl-3 : 4-dihydroquinazoline $(A)$ did not appear satisfactory to us on the basis of the evidence submitted. This constitution is that of the carbinol base of an alkylquinazolinium salt, and it would be highly surprising if such a substance could, like vasicine, be converted into a volatile chlorodeoxybase or acetyl derivative. It was scarcely more credible that such a carbinol base would form normal salts $B, \mathrm{HCl}$ instead of the usual $B, \mathrm{HCl}-\mathrm{H}_{2} \mathrm{O}$, and yet many vasicine salts of the first-cited form have been described. Admittedly these considerations are not infallible guides, and substances do occasionally exhibit totally unexpected properties; fortunately, however, the validity of the Spath-Nikawitz suggestion can be quickly tested experimentally, because the synthesis of $(A)$ may be effected without difficulty.

Allyl iodide and quinazoline combine with formation of 3-allylquinazolinium iodide and this salt (or the corresponding chloride), reacting with alkalis in aqueous solution, yields the carbinol base $(A)$, which crystallises from benzene in well-formed colourless prisms, m.p. $130^{\circ}$ (vasicine, m.p. $208^{\circ}-210^{\circ}$ ) (Found : $\mathrm{C}, 70 \cdot 4 ; \mathrm{H}, 6 \cdot 4 ; \mathrm{N}, 14 \cdot 6 . \quad \mathrm{C}_{11} \mathrm{H}_{12} \mathrm{ON}_{2}$ requires C, $70.2 ; \mathrm{H}, 6.4 ; \mathrm{N}, 14.9$ per cent).

A further description of this and analogous bases will, it is hoped, be published in another place.

It is apparent that vasicine cannot be correctly formulated in accordance with the proposal of Spath and Nikawitz. Of the alternative formulæ rejected by these authors in the course of their discussion, that numbered VI (loc. cit., p. 48) seems to fit the facts best, but it is not wholly satisfactory for several reasons including the optical inactivity of vasicine.

The final sentence of the memoir of Spath and Kuffner is the following: "Uber Einzelheiten der Vasicin-Literatur wollen wir gegenwärtig nicht das Wort ergreifen, sondern die weiteren Ergebnisse der indischen Forscher abwarten." May we be allowed to follow this excellent example, bracketing, however, our Austrian with our Indian colleagues,

\section{T. M. Reynolds,}

R. Robinson.

Dyson Perrins Laboratory, University, Oxford. June 26.

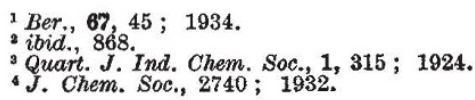

\section{Synthesis of Vitamin $\mathrm{C}$ by the Infant}

THE presence of vitamin $\mathrm{C}$ in human urine has been recently shown both by tests with the Bezssonoff reagent and dichlorphenol-indophenol ${ }^{1}$ and also demonstrated by animal experimentation ${ }^{2}$. We designate as U.H. the unit of violet coloration produced in 1 c.c. of water by $1 / 1,000 \mathrm{mgm}$. of hydroquinone, in the presence of the Bezssonoff reagent $\left(\mathrm{MoO}_{3}\right)\left(\mathrm{WO}_{3}\right)_{17}\left(\mathrm{P}_{2} \mathrm{O}_{5}\right)\left(\mathrm{H}_{2} \mathrm{O}\right)_{24}$. A solution of pure vitamin $\mathrm{C}, \mathrm{N} / \mathbf{1 0 0 , 0 0 0}$, gives a coloration of 1 U.H. This was verified with samples of ascorbic acid received from Micheel, Szent-Gyorgyi and Reichstein. We have actually tested, by this reagent, the urine of infants of 2-23 months in age, submitted during 48 hours to a diet deprived of vitamin $\mathrm{C}$. The following are the results (Fig. 1):

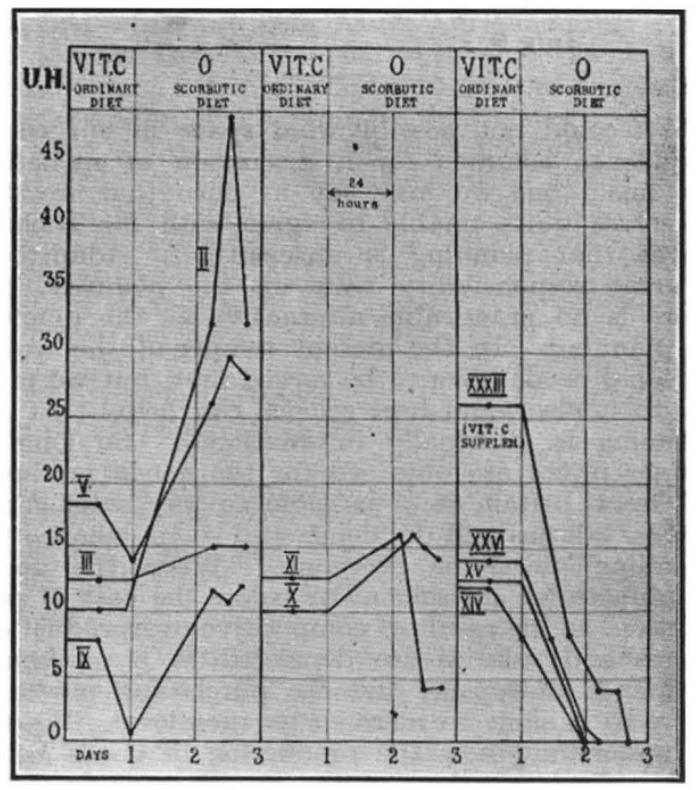

Fig. 1.

The curves indicate the variation of amount of vitamin $\mathbf{C}$ in the urine; the age of the infants, in months, is given by Roman numerals. 